\title{
Unternehmensengagement im deutschen Bildungssystem
}

\section{Zwischen strategischen Unternehmensinteressen und persönlichen Präferenzen}

Peter Friedrich

Peter Friedrich ist wissenschaftlicher Mitarbeiter im Forschungsprojekt »Gesellschaftliches Engagement von Unternehmen in Deutschland « an der Martin-Luther-Universität HalleWittenberg.

E-Mail peter.friedrich@paedagogik.uni-halle.de
Das Bildungssystem gilt als einer der letzten staatlich dominierten Gesellschaftsbereiche. Doch immer öfter kommt es zu Kooperationen zwischen Wirtschaftsunternehmen und Bildungseinrichtungen. Vorbild könnte dabei das duale Ausbildungssystem sein, in dem es schon immer ein Zusammenwirken von Betrieben und Berufsschulen gab.

Seit einigen Jahren wird in Deutschland eine intensive Debatte über das gesellschaftliche Engagement von Unternehmen geführt, die sich durch eine begriffliche und inhaltliche Pluralität auszeichnet (vgl. Schäffer 2004: 4 ff.). So belegen unterschiedliche Untersuchungen eine lange Tradition des gesellschaftlichen Engagements von Unternehmen in Deutschland und verweisen zugleich auf eine Entwicklung des Phänomens im Sinne eines Wandels von einem spontan, zufällig geprägten hin zu einem strategischen und rationalen, am Kerngeschäft ausgerichteten gesellschaftlichen Engagement von Unternehmen (vgl. Braun/Kuckuck 2007, Bertelsmann Stiftung 2005).

Im Rahmen dieser Entwicklung sind ebenfalls die Erschließung neuer sowie der Ausbau etablierter Engagementbereiche $\mathrm{zu}$ beobachten. Hierbei ist das Bildungssystem, mit seinen öffentlichen Schulen und staatlichen Universitäten, bisher nicht zu den klassischen Bereichen des gesellschaftlichen Engagements von Unternehmen zu zählen. Vielmehr oblag die hoheitliche Steuerung des Bildungssystems dem Staat. Ein Sonderfall stellt das duale Ausbildungssystem dar, das seit jeher durch eine intensive Kooperation von staatlichen Berufsschulen und Unternehmen geprägt ist. Gleichwohl gewinnt dieser Engagementbereich zunehmend an gesellschaftlicher Bedeutung. So verweisen unterschiedliche Umfragen auf das Bildungssystem als zukunftsträchtigen Engagementbereich für Unternehmen (FWA 2007, PLEON 2007; 23). Im Fol- genden soll diskutiert werden, inwieweit Gründe für ein gesellschaftliches Engagement der Unternehmen im Bildungsbereich erkennbar sind und welche Beobachtungen bezüglich der Realisierung dieses Engagements beschrieben werden können.

Das Bildungssystem gilt als einer der letzten staatlich dominierten Gesellschaftsbereiche. Insofern besteht ein deutliches Abhängigkeitsverhältnis zum politischen System, in Form der jeweiligen Bundesländer und dem Bildungssystem, das sich durch eine weitreichende Regulierung der Struktur und der Form des Bildungssystems auszeichnet. Gleichzeitig ist das Bildungssystem aktuell durch scheinbare Brüche von Institutionalisierungspfaden gekennzeichnet, die am deutlichsten in der Neuverteilung der Aufgaben und Rollen der relevanten Akteure beobachtbar sind. So ist innerhalb des Bildungssystems ein Wandel verschiedener Institutionen zu beobachten, wie beispielsweise die Entwicklung, die unter dem Begriff der Schulöffnung diskutiert wird. Aber auch grundsätzliche Strukturen unterliegen Veränderungsprozessen, wie das Thema der Ganztagsschulen (vgl. Holtappel) oder Standardisierung (vgl. Leuze/Martens/Rusconi 2007: 3 ff.) zeigt.

Das Verhältnis von Bildungs- und Wirtschaftssystem lässt sich historisch betrachtet, durch wechselseitige Leistungserwartungen beschreiben (vgl. Eichmann 1989: 82). An dieser Stelle ist besonders auf die Leistungserwartungen seitens des Wirtschaftssystems gegenüber dem Bildungssystem hinzuweisen, die häufig Gegenstand der öffentlichen Debatte sind. Die Leistungserwartungen beziehen sich vorrangig auf die Ausbildung qualifizierter Absolventen und deren Vorbereitung auf die Arbeitswelt (vgl. Fend 2006: 37 f., Eichmann 1989: 73 f.). Sie sind verbunden mit der Forderung, dass das Bildungssystem seine "gesellschaftlichen Aufgaben « möglichst effizient und quali- 
tativ hochwertig erfüllen soll (vgl. u. a VBW 2005, BDA 2003). Konkret wird seitens des Wirtschaftssystems auf den mangelnden Arbeitsweltbezug, das sinkende schulische Leistungsniveau sowie die zeitlich verzögerte Anpassung an veränderte Umweltbedingungen und Umwelterwartungen hingewiesen (vgl. OECD/CERI 1995: 28 ff.).

Daraus lässt sich eine kritische Betrachtung speziell durch die Unternehmerinnen und Unternehmer sowie der Unternehmensverbände gegenüber dem deutschen Bildungssystem ableiten. Dies wird ebenfalls anhand der Fülle von Positionspapieren von Wirtschaftsvertretern zum Thema Bildung deutlich (siehe u. a. VBW 2005, BDA 2003). Basierend auf dieser defizito- nehmen zu: »Die Schulen haben halt immer Angst, die Unternehmen kommen hier rein und verkaufen nur ihr Zeug an die Schüler, ob das Süßigkeiten oder Softwareprogramme sind, sei erst einmal dahingestellt. Und die Unternehmen haben immer den Vorbehalt, das ist alles irgendwie unprofessionell und damit können wir nicht. « vgl. Friedrich 2007: 4)

Neben den traditionellen Engagementformen des Spenden und Sponsorings ist eine häufig vorzufindende Form des gesellschaftlichen Engagements von Unternehmen die Kooperation mit unterschiedlichen Organisationen. An dieser Stelle ist beispielsweise auf die Vielzahl von universitären Lehrstühlen, die von Unternehmensstiftungen finanziert werden, oder

\section{"Kooperationen von Organisationen des Bildungssystems und Unternehmen werden oft durch wechselseitige Vorurteile behindert «}

rientierten Perspektive kann ein gesellschaftliches Engagement von Unternehmen im Bereich der Bildung als Versuch der Sicherung und Wahrung eigener Leistungserwartungen gegenüber dem Bildungssystem angesehen werden, deren Erfüllung ohne dieses Engagement unwahrscheinlich wäre (vgl. Friedrich 2007: 38 ff.).

Diesem Ansatz folgend deutet sich seitens der Unternehmen ein reaktives Engagement an, dass sich jedoch nicht wie bei anderen Engagementthemen auf Forderungen der Unternehmensumwelt bezieht, sondern die Sicherung eigener Interessen verfolgt, im Sinne von Leistungserwartungen gegenüber dem Bildungssystem. Somit sind neben einem Mehrwert für das Bildungssystem auch deutlich unternehmensstrategischen Überlegungen zu nennen.

Derartige Konstellation von Organisationen des Bildungssystems und sich engagierenden Unternehmen erscheinen hoch voraussetzungsvoll, da zum einen unterschiedliche Handlungslogiken aufeinandertreffen und zum anderen diese Konstellation historisch durch wechselseitige Vorurteile belastet zu sein scheint. So spitzt es eine Führungsperson eines Unter- auf die Schülerfirmen und Wirtschaftsprojekte in Schulen sowie durch Unternehmen geförderte Wettbewerbe hinzuweisen. Gleichwohl einer zu beobachtenden Mittelfristigkeit dieser Kooperationsbeziehungen ist eine strategische Ausrichtung, wie sie von Unternehmen beschrieben und in der aktuellen Diskussion um das gesellschaftliche Engagement von Unternehmen eingefordert wird, nur bedingt erkennbar. So wirken die beschriebenen unternehmensstrategischen Überlegungen im Sinne einer Einbindung des gesellschaftlichen Engagements in die Unternehmensstrategie als bedingt realisierbar. Vielmehr scheint das gesellschaftliche Engagement als zufällig und spontan, auf persönlichen Beziehungen basierend (vgl. Friedrich 2007: 100 f.). So ist einzelnen Personen und ihren Rollen innerhalb der Organisationen in Bezug auf die Auswahl und Bewertung der Kooperationen eine hohe Bedeutung zuzumessen. Oder wie es Gail Niedernhorfer formuliert: »Die meisten erfolgreichen Partnerschaften sind davon abhängig, dass eine irrational engagierte Einzelperson sie mit starker Hand von oben lenkt. «(OECD/CERI 1995: 20)
Die Entscheidungsträger der Organisationen stellen als Personen wichtige Bindeglieder für die beschriebenen Kooperationen dar, indem sie durch persönliche vertrauensbasierte Entscheidungen eine Alternative zu rationalen und messbaren Entscheidungen bieten und auf diese Weise das gesellschaftliche Engagement als Handeln ihrer Organisation legitimieren. Oder zugespitzt formuliert: »Alles was nicht messbar ist, ist auch nicht $\mathrm{zu}$ managen! « (Bluszcz 2007: 107). Dieser Aussage folgend, setzt ein unternehmensstrategisches gesellschaftliches Engagement die Messbarkeit desselben voraus. Eine Alternative dazu stellen personalisierte Entscheidungen von Führungs- und Leitungspersonen dar, die durch persönliche Präferenzen Entscheidungen ermöglichen. Dieser Argumentation folgend, erscheint das strategische gesellschaftliche Engagement von Unternehmen im Bildungsbereich als anspruchsvoll. Vielmehr deutet sich an, dass eine Etablierung dieses im Rahmen von persönlichen Präferenzen der Leitungs- und Führungspersonen innerhalb der Unternehmen erfolgt und erst dadurch Priorität im Unternehmen genießt.

Die bisherige Argumentation blieb auf ein duales Beziehungsverhältnis von Wirtschafts- und Bildungssystem beschränkt. Gleichwohl ist die gesellschaftliche Einbettung dieser Beziehung zu berücksichtigen. Hierbei kommt »dem « Staat - konkret den jeweiligen Bundesländern - eine besondere Rolle zu, denen die Steuerungshoheit des Bildungssystems obliegt. Aber auch andere Akteure gewinnen im Rahmen eines sich öffnenden Bildungssystems an gesellschaftspolitscher Bedeutung. Somit handelt es sich um eine Neuverteilung von gesellschaftlichen Aufgaben und Funktion innerhalb der Gesellschaft (vgl. Backhaus-Maul 2004: 26).

Wie angedeutet, ist das Bildungssystem, das durch ein Abhängigkeitsverhältnis zu dem politischen System beschrieben werden kann, ebenfalls durch scheinbare Brüche von Institutionalisierungspfaden gekennzeichnet. Für diesen Trend können unterschiedliche Lesarten präferiert werden. Dem skizzierten Motiv folgend, kann das gesellschaftliche Engagement von Unternehmen im Bildungsbereich als Kompensierung von wahrgenommenen Defiziten betrachtet werden (vgl. Ries 1971: 106). An dieser Stelle ist gleichzeitig zu hinterfragen, inwieweit das 
gesellschaftliche Engagement von Unternehmen als Kompensationsform der artikulierten Defizite geeignet zu sein scheint.

Des Weiteren ist offen, inwieweit es institutionalisiert wird oder weiterhin als zufälliges und spontanes Artefakt persönlicher Präferenzen beobachtbar ist. Besondere Bedeutung scheint diesbezüglich den staatlichen Rahmenbedingungen zuzukommen, die auf zukünftige Kooperationen zwischen Unternehmen und Organisationen des Bildungssystems prägenden Einfluss haben werden. Da die staatliche Steuerung sowie deren potenzieller Rückzug für die beschriebenen Kooperationsbeziehungen den Handlungsrahmen darstellt. Zugleich ist weiterhin zu hinterfragen, inwieweit die Umwelt dieser Kooperationsbeziehungen - beispielsweise in Form von Eltern und NonProfit-Organisationen - für die Realisierung dieser relevant erscheint.

\section{Engagiert unternehmen}

Im Schnitt wurden in Deutschlands Unternehmen im Jahr 2000 je 100.000 Euro Umsatz freiwillig etwa 70 Euro in Soziales investiert, wobei kleine und mittlere Unternehmen relativ mehr ausgaben als die Großen.

Der Paritätische Wohlfahrtsverband in Nordrhein-Westfalen hat deshalb das Buch »Engagiert unternehmen « vorgelegt. Darin werden Praxisbeispiele über die Zusammenarbeit sozialer Initiativen mit Wirtschaftsunternehmen vorgestellt. Die Kooperationen verlaufen meist erfolgreich, mitunter aber auch mit Problemen wie das Beispiel Selbsthilfe und Pharmaindustrie zeigt. Auf über 120 Seiten stellt das Buch konkrete Beispiele aus der Zusammenarbeit vor und spricht praxisbezogen deren Chancen und Risiken an. Zugleich eröffnet »Engagiert unternehmen « die Perspektive einer strategischen Allianz dieser unterschiedlichen Partner, die doch gemeinsame Akteure in einem neuen Modell einer sozialen Zivilgesellschaft sein können.

Das Buch »Engagiert unternehmen « kostet 12,50 Euro zuzüglich Versandkosten (Sonderregelung für Mitgliedsorganisationen des Paritätischen Wohlfahrtsverbandes).

Der Paritätische Wohlfahrtsverband, Landesverband Nordrhein-Westfalen e.V., Loher Straße 7, 42283 Wuppertal, Telefon 0202 2822-0, Internet http://www.paritaet-nrw.org

\section{Literatur}

Backhaus-Maul, Holger (2004): Corporate Citizenship im deutschen Sozialstaat. In: Aus Politik und Zeitgeschichte. Heft 14. S. 23-30.

Bundesvereinigung der Deutschen Arbeitgeberverbände BDA (2005): Master of Education. Für eine neue Lehrerausbildung. Berlin.

Bertelsmann Stiftung (2005): Die gesellschaftliche Verantwortung von Unternehmen.

Bluszcz, Oliver (2007): Strategische Allianzen zwischen Profit und Non-Profit-Organisationen. In: Hafner, Sonja F./Hartel, Jörg/ ders./Stark, Wolfgang (Hg.) (2007): Gesellschaftliche Verantwortung in Organisationen. München. S. 107-118.

Braun, Sebastian/Kuckuck, Marc (2007): Corporate Citizenship: Gesellschaftliches Engagement von Wirtschaftsunternehmen in Deutschland. Kommentierter Datenbericht zum Forschungsprojekt, in: Universität Paderborn/Forschungszentrum für Bürgerschaftliches Engagement (Hg.): Working Paper Nr. 1/2007. Paderborn.

Eichmann, Rainer (1989): Diskurs gesellschaftlicher Teilsystem. Zur Abstimmung von Bildungssystem und Beschäftigungssystem. Wiesbaden.

Fend, Helmut (2006): Neue Theorie der Schule. Einführung in das Verstehen von Bildungssystemen. Wiesbaden.

Freiwilligenagentur Halle-Saalkreis e. V. FWA (Hrsg.) (2007): Praxishandbuch »Corporate Citizenship «- gemeinsam engagiert. Erfahrungen, Strategien und Modelle für gesellschaftliches Engagement von Unternehmen in Mitteldeutschland. Halle.

Friedrich, Peter (2007): Kooperation von öffentlichen Schulen und Wirtschaftsunternehmen. Zwischen strategischem Engagement, systemorientiertem Einfluss und Zufall. Halle: Martin-Luther-Universität/Philosophische Fakultät III.

Hafner, Sonja (2007): Strategien und Wandel bei Trendsettern gesellschaftlicher Verantwortung: Über die treibenden Kräfte und Effekte im Innovations-ImitationProzess. In: . dies./Hartel, Jörg/Bluszcz, Oliver/Stark, Wolfgang (Hg.) (2007): Gesellschaftliche Verantwortung in Organisationen. München. S. 73-92.

Holtappels, Heinz Günter (1994): Ganztagsschule und Schulöffnung. Perspektiven für die Schulentwicklung. Weinheim.

Martens, Kerstin/Rusconi, Alessandra/Leuze, Kathrin (2007): New Arenas of Education. The Impact of International Organizations and Markets on Education Policy Making.. Basingstoke, Hampshire.

OECD/CERI (1995): Schule und Wirtschaft: eine neue Partnerschaft. Frankfurt am Main.

PLEON (Hg.) (2007): Sponsoring Trends 2006.

Ries, Heinz (1971): Soziale Struktur des Bildungssystems und Sozialisation von Talenten. Stuttgart.

Schäfer, Henry (2004): Transparenzstudie. zur Beschreibung ausgewählter international verbreiteter Rating-Systeme zur Erfassung von Corporate Social Responsibility. Hg. von der Bertelsmann Stiftung. Gütersloh.

vbw - Vereinigung der Bayrischen Wirtschaft e. V. (2005): Bildung neu denken! Das juristische Konzept. Wiesbaden. 